\title{
Standard and Corrected Numerical Differentiation Formulae
}

\author{
François Dubeau*
}

\begin{abstract}
Standard numerical differentiation rules that might be established by the method of undetermined coefficients are revisited. Best truncation error bounds are established by a direct method and by the method of integration by parts "backwards". A new method to increase the order of the truncation error using a primitive is presented. This approach leads to corrected numerical differentiation rules. Differentiation formulae and numerical tests are presented.

Keywords: Absolutely continuous function, Method of undetermined coefficients, Numerical differentiation rules, Peano kernel, Taylor's expansion.

2010 AMS: Primary 65D25, Secondary 26A24

Départment de Mathématiques, Faculté des sciences, Université de Sherbrooke, Sherbrooke (Qc), Canada, ORCID: 0000000229563208 *Corresponding author: francois.dubeau@usherbrooke.ca

Received: 14 January 2019, Accepted: 12 April 2019, Available online: 27 June 2019
\end{abstract}

\section{Introduction}

Many textbooks of numerical analysis present the method of undetermined coefficients to find and approximation of the integral or the derivative of a given function, for example [1, 2, 3, 4] and many others. The method of undetermined coefficients used to estimate the $k$-th derivative $f^{(k)}(0)$ of a given function $f(x)$ consists in finding a $(n+1)$-dimensional weight vector $\vec{a}=\left(a_{0}, \ldots, a_{n}\right)$ associated to a given $(n+1)$-dimensional vector of distinct coordinates (or nodes) $\vec{x}=\left(x_{0}, \ldots, x_{n}\right)$ with $n \geq k$ and $\left|x_{i}\right| \leq 1$ for all $i$, such that the quantity $D^{(k)}(f ; h)$ given by the formula

$$
f^{(k)}(0) \approx \frac{1}{h^{k}} \sum_{i=0}^{n} a_{i} f\left(h x_{i}\right) .
$$

The method of undetermined coefficients is based on the requirement that the truncation error

$$
R_{D^{(k)}}(f ; h)=h^{k} f^{(k)}(0)-\sum_{i=0}^{n} a_{i} f\left(h x_{i}\right)
$$

be such that

$$
R_{D^{(k)}}(f ; h)=o\left(h^{r(n)}\right)
$$

where $r(n) \geq n$ depends on the regularity of $f(x)$. 
It is possible to increase the order of the truncation error term. Indeed, if a primitive $F(x)$ is available, that is to say $F^{\prime}(x)=f(x)$, we can add an expression to the preceding approximation as follows

$$
f^{(k)}(0) \approx \frac{1}{h^{k}}\left(\sum_{i=0}^{n} a_{i} f\left(h x_{i}\right)+c\left[\frac{1}{h} \sum_{j=0}^{m} \beta_{j} F\left(h \xi_{j}\right)-\sum_{i=0}^{n} b_{i} f\left(h x_{i}\right)\right]\right),
$$

where $(n+1)$-dimensional weight vector $\vec{b}=\left(b_{0}, \ldots, b_{n}\right)$, the two $(m+1)$-dimensional vectors of weight $\vec{\beta}=\left(\beta_{0}, \ldots, \beta_{m}\right)$ and distinct coordinates (or nodes) $\vec{\xi}=\left(\xi_{0}, \ldots, \xi_{m}\right)$, and c are chosen in such a way that the truncation error given by

$$
R_{D^{(k)}}^{c}(f ; h)=h^{k} f^{(k)}(0)-\sum_{i=0}^{n} a_{i} f\left(h x_{i}\right)-c\left[\frac{1}{h} \sum_{j=0}^{m} \beta_{j} F\left(h \xi_{j}\right)-\sum_{i=0}^{n} b_{i} f\left(h x_{i}\right)\right],
$$

is such that

$$
R_{D^{(k)}}^{c}(f ; h)=o\left(h^{r^{c}(n)}\right),
$$

with $r^{c}(n)>r(n)$.

The plan of the paper is the following. In the next section, we present preliminaries about polynomials, Vandermonde matrix, and Taylor's expansions. Section 3 presents the standard approach for obtaining differentiation rules using the method of undetermined coefficients. We establish optimal truncation error bounds by a direct approach and by the method of integration by parts "backward". Total error bound composed of the truncation term and of the roundoff error term is given. In Section 4, we present a method to improve the error by adding information coming from a primitive. Examples of formula are given in Section 5 and numerical tests are included in Section 6.

We will use $f^{(l)}(x)$ for the $l$-th derivative of $f(x)$ for $l=0,1,2, \ldots$, and $f^{(0)}(x)=f(x)$. Let $1 \leq p \leq \infty$, if $f(x)$ is defined on a set $E,\|f\|_{p, E}$ will be its $p$-norm on $E$, and if $\vec{v}$ is a vector in $\mathbb{R}^{n}$, its $p$-norm will be $\|\vec{v}\|_{p}$.

\section{Preliminaries}

\subsection{Small $O$ and big $O$ notations}

Let $f(x)$ be a function such that $\lim _{x \rightarrow \alpha} f(x)=0$. We say that $g(x)$ is a small $o$ of $f(x)$ around $\alpha$, and write $g(x)=o(f(x))$, if for any $\varepsilon>0$ there exists a $\delta_{\varepsilon}>0$ such that

$$
|g(x)| \leq \varepsilon|f(x)| .
$$

holds for $0<|x-\alpha|<\delta_{\varepsilon}$. We say that $g(x)$ is a big $O$ of $f(x)$ around $\alpha$, and write $g(x)=O(f(x))$, if there exist a constant $C$ and a $\delta>0$ such that

$$
|g(x)| \leq C|f(x)| .
$$

holds for $0<|x-\alpha|<\delta$.

Lemma 2.1. Let us assume that the real number $r>0$ and $n=\lfloor r\rfloor \geq 0$. Let $\pi_{m}(x)$ be a polynomial of degree $m$ such that

$$
\pi_{m}(x)=o\left(|x-\alpha|^{r}\right) .
$$

Then,

$$
\pi_{m}(x)=\left\{\begin{array}{lll}
(x-\alpha)^{n} \pi_{m-n}(x) & \text { if } & m>r \\
0 & \text { if } & m \leq r,
\end{array}\right.
$$

where $\pi_{m-n}(x)$ is a polynomial of degree $m-n$.

\subsection{Vandermonde matrix and Lagrange interpolation polynomials}

Let $\vec{x}=\left(x_{0}, \ldots, x_{n}\right)$ bet a $n+1$-vector of distincts real (or complex) numbers and its associated Vandermonde matrix $V(\vec{x})$,

$$
V(\vec{x})=\left[\begin{array}{ccc}
1 & \ldots & 1 \\
x_{0} & & x_{n} \\
\vdots & & \vdots \\
x_{0}^{n} & \ldots & x_{n}^{n}
\end{array}\right] .
$$


Let $\vec{e}_{l}$ be the $(n+1)$-column vector, the transpose of $\left(\delta_{l, 0}, \cdots, \delta_{l, j} \cdots, \delta_{l, n}\right)$, where

$$
\delta_{l, j}=\left\{\begin{array}{c}
1 \quad \text { if } \quad j=l, \\
0 \text { if } j \neq l,
\end{array}\right.
$$

for $0 \leq l, j \leq n$.

Lemma 2.2. [5] The Vandermonde matrix $V(\vec{x})$ is invertible and the l-th column of $V^{-1}(\vec{x})$ is

$$
V^{-1}(\vec{x}) \vec{e}_{l}=\frac{1}{l !}\left[\begin{array}{c}
w_{n, 0}^{(l)}(0) \\
w_{n, 1}^{(l)}(0) \\
\vdots \\
w_{n, n}^{(l)}(0)
\end{array}\right],
$$

for $l=0, \ldots, n$, where $\left\{w_{n, j}(x)\right\}_{j=0}^{n}$ is the Lagrange's basis of the space of polynomial of degree at most $n$.

\subsection{Taylor's expansion}

Let $I_{h}=[-h, h], I_{h}^{+}=[0, h]$ and $I_{h}^{-}=[-h, 0]$. For $h=1$ we will simply use $I=[-1,1], I^{+}=[0,1]$ and $I^{-}=[-1,0]$. Let $p$ and $q$ be two real numbers such that $1 \leq p, q \leq \infty$ and $\frac{1}{p}+\frac{1}{q}=1$. Let $C^{l}\left(I_{h}\right)$ be the set of continuously differentiable functions up to order $l$ on $I_{h}$, and the set of absolutely continuous function on $I_{h}$ be defined by

$$
A C^{l+1, p}\left(I_{h}\right)=\left\{\begin{array}{l|l}
f \in C^{l}\left(I_{h}\right) \mid \begin{array}{l}
(a) f^{(l+1)} \in L^{p}\left(I_{h}\right), \text { and } \\
(b) f^{(l)}(s)=f^{(l)}(r)+\int_{r}^{s} f^{(l+1)}(\xi) d \xi, \forall r, s \in I_{h}
\end{array}
\end{array}\right\} .
$$

Taylor's expansion of $f(x) \in A C^{l+1, p}\left(I_{h}\right)$ around $x=0$ of order $l+1$ is

$$
f(x)=\sum_{j=0}^{l} \frac{f^{(j)}(0)}{j !} x^{j}+\int_{-h}^{h} f^{(l+1)}(y) K_{T, l}(x, y ; h) d y .
$$

where $K_{T, l}(x, y ; h)$ is the kernel

$$
K_{T, l}(x, y ; h)=\frac{1}{l !}\left[(x-y)_{+}^{l} \mathbf{1}_{I_{h}^{+}}(y)+(-1)^{l+1}(y-x)_{+}^{l} \mathbf{1}_{I_{h}^{-}}(y)\right],
$$

for any $x, y$ in $I_{h}[6,7]$. This kernel is a piecewise polynomial function of degree $l$. In this expression, if $E$ is a set, then

$$
\mathbf{1}_{E}(y)=\left\{\begin{array}{lll}
1 & \text { if } & y \in E, \\
0 & \text { if } & y \notin E .
\end{array}\right.
$$

Also for any $l \geq 0,(\eta)_{+}^{l}$ is defined

$$
(\eta)_{+}^{l}=\eta^{l} \mathbf{1}_{(0,+\infty)}(\eta) .
$$

If we set $x=h \xi$, and $y=h \eta$, then the kernel becomes

$$
K_{T, l}(x, y ; h)=K_{T, l}(h \xi, h \eta ; h)=h^{l} K_{T, l}(\xi, \eta ; 1),
$$

for any $\xi, \eta$ in $I$.

\section{Standard numerical differentiation rules}

\subsection{Existence: method of undetermined coefficients}

Let us observe that $R_{D^{(k)}}(f ; h)$ is linear expression with respect to $f(x)$ and also if $f(x)$ is a polynomial of degree $\leq m$ with respect to $x$, then $R_{D^{(k)}}(f ; h)$ is a polynomial of degree $\leq m$ with respect to $h$. The condition (1.2), combined to Lemma 2.1, 
implies that $R_{D^{(k)}}(f ; h)=0$ for any polynomial $f(x)$ of degree $\leq n$. So using the standard basis $\left\{x^{l}\right\}_{l=0}^{n}$, we have to solve the linear system

$$
\sum_{i=0}^{n} a_{i} x_{i}^{l}=\frac{D^{(k)}\left(x^{l} ; h\right)}{h^{l}}=k ! \delta_{k, l} \quad \text { for } \quad l=0, \ldots, n,
$$

for which the solution is

$$
\vec{a}=k ! V^{-1}(\vec{x}) \vec{e}_{k}=\left[\begin{array}{c}
w_{n, 0}^{(k)}(0) \\
w_{n, 1}^{(k)}(0) \\
\vdots \\
w_{n, n}^{(k)}(0)
\end{array}\right] .
$$

We obtain the method

$$
f^{(k)}(0) \approx \frac{\sum_{i=0}^{n} a_{i} f\left(h x_{i}\right)}{h^{k}} .
$$

It might happen that $R_{D^{(k)}}(f ; h)=0$ for some polynomials of degree $k>n$. Let us define the degree of accuracy (or precision) $k_{a}$ of the approximation process (1.1) to be the largest integer $k_{a} \geq n$ such that $R_{D^{(k)}}(f ; h)=0$ holds for any polynomial $f(x)$ of degree $l \leq k_{a}$.

\subsection{Truncation error}

Two different approaches can be used to establish the best bounds for the truncation error in terms of the regularity of $f(x)$. The first approach will be called the standard direct approach, while the second way is the integration by parts "backwards". This second approach presented in $[8,9,10]$, usually presented for numerical integration [11], can be used in general when we consider the method of undetermined coefficients [12]. Let us note some bounds were already presented for specific formulae elsewhere, for example in [13].

\subsubsection{Direct approach}

For any integer $l$ such that $k \leq l \leq k_{a}$, let $f(x) \in A C^{l+1, p}\left(I_{h}\right)$. Since the process is exact for polynomials of degree $\leq l$, using a Taylor's expansion of order $l+1$, the truncation error is

$$
R_{D^{(k)}}(f ; h)=\int_{-h}^{h} f^{(l+1)}(y) K_{D^{(k), l}}(y ; h) d y,
$$

where $K_{D^{(k)}, l}(y ; h)$ is the Peano kernel associated to the process given by

$$
\begin{aligned}
K_{D^{(k), l}}(y ; h) & =R_{D^{(k)}}\left(K_{T, l}(\cdot, y ; h) ; h\right) \\
& =h^{k} K_{T, l-k}(0, y ; h)-\sum_{i=0}^{n} a_{i} K_{T, l}\left(h x_{i}, y ; h\right) .
\end{aligned}
$$

It follows that

$$
\left|R_{D^{(k)}}(f ; h)\right| \leq\left\|f^{(l+1)}\right\|_{p, I_{h}}\left\|K_{D^{(k), l}(\cdot ; h)}\right\|_{q, I_{h}} .
$$

Moreover, $K_{D^{(k),},}(y ; h)=K_{D^{(k)}, l}(h \eta ; h)=h^{l} K_{D^{(k)}, l}(\eta ; 1)$, then

$$
\left\|K_{D^{(k)}, l}(\cdot ; h)\right\|_{q, I_{h}}=h^{l+1-\frac{1}{p}}\left\|K_{D^{(k), l}}(\cdot ; 1)\right\|_{q, I} .
$$

So

$$
\left|R_{D^{(k)}}(f ; h)\right| \leq h^{l+1-\frac{1}{p}} C_{k ; l, p}\left\|f^{(l+1)}\right\|_{p, I_{h}},
$$

where

$$
C_{k ; l, p}=\left\|K_{D^{(k), l},(\cdot ; 1)}\right\|_{q, I},
$$


does not depend on $h$. Since because

$$
\lim _{h \rightarrow 0}\left\|f^{(l+1)}\right\|_{p, I_{h}}=\left\{\begin{array}{lll}
0 & \text { for } & 1 \leq p<\infty, \\
C & \text { for } & p=\infty
\end{array}\right.
$$

we have

$$
R_{D^{(k)}}(f ; h)= \begin{cases}o\left(h^{l+1-\frac{1}{p}}\right) & \text { for } 1 \leq p<\infty, \\ O\left(h^{l+1}\right) & \text { for } \quad p=\infty .\end{cases}
$$

Since an $o\left(h^{l+1-\frac{1}{p}}\right)$ and an $O\left(h^{l+1}\right)$ are $o\left(h^{l}\right)$, it means that $R_{D^{(k)}}(f ; h)=o\left(h^{l}\right)$. In summary we have proved the following theorem which presents necessary and sufficient conditions to obtain the desired error order.

Theorem 3.1. For any $l$ such that $n \leq l \leq k_{a}$, a necessary and sufficient condition to have $R_{D^{(k)}}(f ; h)=o\left(h^{l}\right)$ for any $f \in A C^{l+1, p}\left(I_{h}\right)$ is that $R_{D^{(k)}}(f ; h)=0$ for any polynomial $f(x)$ of degree $\leq l$.

Theorem 3.2. If $R_{D^{(k)}}(f ; h)=0$ for any polynomial of degree $\leq k_{a}$, then (3.2) and (3.3) hold for any $f \in A C^{l+1, p}\left(I_{h}\right)$ for $k \leq l \leq k_{a}$.

Remark 3.3. The bounds given by (3.2) and (3.3) are the best one as it has been shown in the general case of the method of undetermined coefficient [12].

Remark 3.4. Let us specify the kernels $K_{D^{(k), l}}(y ; h)$ and $K_{D^{(k), l}}(\eta ; 1)$.

$$
\begin{aligned}
K_{D^{(k), l}}(y ; h)= & h^{k} K_{T, l-k}(x, y ; h)-\sum_{i=0}^{n} a_{i} K_{T, l}\left(h x_{i}, y ; h\right) \\
= & \frac{h^{k}}{(l-k) !}\left[(0-y)_{+}^{l-k} \boldsymbol{I}_{I_{h}^{+}}(y)+(-1)^{l-k+1}(y-0)_{+}^{l-k} \boldsymbol{1}_{I_{h}^{-}}(y)\right] \\
& -\frac{1}{l !} \sum_{i=0}^{n} a_{i}\left[\left(h x_{i}-y\right)_{+}^{l} \boldsymbol{I}_{I_{h}^{+}}(y)+(-1)^{l+1}\left(y-h x_{i}\right)_{+}^{l} \boldsymbol{I}_{I_{h}^{-}}(y)\right],
\end{aligned}
$$

and

$$
K_{D^{(k), l}}(\eta ; 1)=-\frac{1}{l !} \sum_{i=0}^{n} a_{i}\left[\left(x_{i}-\eta\right)_{+}^{l} \mathbf{1}_{I^{+}}(\eta)+(-1)^{l+1}\left(\eta-x_{i}\right)_{+}^{l} \mathbf{1}_{I^{-}}(\eta)\right] .
$$

\subsubsection{Integration by parts "backwards"}

The method of integration by parts "backwards" is based on the Taylor's expansion of

$$
W_{D^{(k)}}(f ; h)=h R_{D^{(k)}}(f ; h) .
$$

We suppose that $f \in A C^{l+1, p}\left(I_{h}\right)$ for $k \leq l \leq k_{a}$, and we proceed as follows. We have

$$
W_{D^{(k)}}(f ; h)=h R_{D^{(k)}}(h ; f)=h^{k+1} f^{(k)}(0)-h \sum_{i=0}^{n} a_{i} f\left(h x_{i}\right),
$$

so $W_{D^{(k)}}(f ; 0)=0$. For $k \leq l \leq k_{a}$ and $1 \leq j \leq l-1$

$$
W_{D^{(k)}}^{(j)}(f ; h)=(k+1){ }_{j} h^{k+1-j} f^{(k)}(0)-j \sum_{i=0}^{n} a_{i} x_{i}^{j-1} f^{(j-1)}\left(h x_{i}\right)-h \sum_{i=0}^{n} a_{i} x_{i}^{j} f^{(j)}\left(h x_{i}\right),
$$

where for two non negative integers $k$ and $j$

$$
(k)_{j}=\left\{\begin{array}{cl}
\frac{k !}{(k-j) !} & \text { for } \quad j=0, \ldots, k \\
0 & \text { for } \quad j>k
\end{array}\right.
$$


Then

$$
\lim _{h \rightarrow 0} W_{D^{(k)}}^{(j)}(f ; h)=(k+1)_{j} \delta_{j, k+1} f^{(k)}(0)-j f^{(j-1)}(0) \sum_{i=0}^{n} a_{i} x_{i}^{j-1}=0 .
$$

Also for $j=l$

$$
W_{D^{(k)}}^{(l)}(f ; h)=(k+1)_{l} h^{k+1-l} f^{(k)}(0)-l \sum_{i=0}^{n} a_{i} x_{i}^{l-1} f^{(l-1)}\left(h x_{i}\right)-h \sum_{i=0}^{n} a_{i} x_{i}^{l} f^{(l)}\left(h x_{i}\right),
$$

and using Taylor's expansions of order 2 for $f^{(l-1)}(x)$ which is in $A C^{2, p}\left(I_{h}\right)$ and of order 1 for $f^{(l)}(x)$ which is in $A C^{1, p}\left(I_{h}\right)$, we obtain

$$
W_{D^{(k)}}^{(l)}(f ; h)=(k+1) h_{l}^{k+1-l} f^{(k)}(0)-l f^{(l-1)}(0) \sum_{i=0}^{n} a_{i} x_{i}^{l-1}-h(l+1) f^{(l)}(0) \sum_{i=0}^{n} a_{i} x_{i}^{l}+\int_{-h}^{h} f^{(l+1)}(y) K_{W, l}(y ; h) d y .
$$

Now, considering (3.1) to simplify, we obtain

$$
W_{D^{(k)}}^{(l)}(f ; h)=\int_{-h}^{h} f^{(l+1)}(y) K_{W, l}(y ; h) d y,
$$

where

$$
K_{W, l}(y ; h)=-l \sum_{i=0}^{n} a_{i} x_{i}^{l-1} K_{T, 1}\left(h x_{i}, y ; h\right)-h \sum_{i=0}^{n} a_{i} x_{i}^{l} K_{T, 0}\left(h x_{i}, y ; h\right) .
$$

Let us remark that $K_{W, l}(y ; h)=h K_{W, l}(\eta ; 1)$ for $y=h \eta$. So the Taylor's expansion of order $l$ for $W_{n}(f ; h)$ leads to

$$
\begin{aligned}
W_{D^{(k)}}(f ; h) & =\int_{0}^{h} W_{D^{(k)}}^{(l)}(f ; z) \frac{(h-z)^{l-1}}{(l-1) !} d z \\
& =\int_{0}^{h}\left[\int_{-z}^{z} f^{(l+1)}(y) K_{W, l}(y ; z) d y\right] \frac{(h-z)^{l-1}}{(l-1) !} d z \\
& =\int_{-h}^{h} f^{(l+1)}(y)\left[\int_{|y|}^{h} K_{W, l}(y ; z) \frac{(h-z)^{l-1}}{(l-1) !} d z\right] d y \\
& =\int_{-h}^{h} f^{(l+1)}(y) \widehat{K}_{D^{(k)}, l}(y ; h) d y,
\end{aligned}
$$

for

$$
\widehat{K}_{D^{(k), l}}(y ; h)=\int_{|y|}^{h} K_{W, l}(y ; z) \frac{(h-z)^{l-1}}{(l-1) !} d z .
$$

As indicated in Remark 3.3, we can obtain the best bound from this expression. So we get the following result.

Theorem 3.5. Let $h>0$, the kernels $K_{D^{(k),}, l}(y ; h)$ and $\widehat{K}_{D^{(k)}, l}(y ; h)$ are such that

$$
h K_{D^{(k), l}}(\cdot ; h)=\widehat{K}_{D^{(k), l}}(\cdot ; h)
$$

almost everywhere.

As a consequence both methods lead to the same best error bounds.

Remark 3.6. The kernel is

$$
\begin{aligned}
K_{W, l}(y ; h)= & -\left[l \sum_{i=0}^{n} a_{i} x_{i}^{l-1}\left(h x_{i}-y\right)_{+}+h \sum_{i=0}^{n} a_{i} x_{i}^{l}\left(h x_{i}-y\right)_{+}^{0}\right] \boldsymbol{I}_{I_{h}^{+}}(y) \\
& -\left[l \sum_{i=0}^{n} a_{i} x_{i}^{l-1}\left(y-h x_{i}\right)_{+}-h \sum_{i=0}^{n} a_{i} x_{i}^{l}\left(y-h x_{i}\right)_{+}^{0}\right] \boldsymbol{I}_{I_{h}^{-}}(y)
\end{aligned}
$$

or, after the substitution $y=h \eta$ and simplification,

$$
\begin{aligned}
K_{W, l}(\eta ; 1)= & -\left[l \sum_{i=0}^{n} a_{i} x_{i}^{l-1}\left(x_{i}-\eta\right)_{+}+\sum_{i=0}^{n} a_{i} x_{i}^{l}\left(x_{i}-\eta\right)_{+}^{0}\right] \mathbf{1}_{I^{+}}(\eta) \\
& -\left[l \sum_{i=0}^{n} a_{i} x_{i}^{l-1}\left(\eta-x_{i}\right)_{+}-\sum_{i=0}^{n} a_{i} x_{i}^{l}\left(\eta-x_{i}\right)_{+}^{0}\right] \mathbf{1}_{I^{-}}(\eta) .
\end{aligned}
$$




\subsection{Total error}

In effective numerical computation, the quantity $f^{(k)}(0)$ is approximated by

$$
\frac{1}{h^{k}} \sum_{i=0}^{n} a_{i} \tilde{f}\left(h x_{i}\right)
$$

which uses $\tilde{f}\left(h x_{i}\right)$ instead of $f\left(h x_{i}\right)$, so introducing roundoff error $\mathrm{e}_{i}=f\left(h x_{i}\right)-\tilde{f}\left(h x_{i}\right)$. The total error $E(f ; h)$ is decomposed in two types of error: the truncation error $R_{D^{(k)}}(f ; h)$ and the roundoff error $S(f ; h)$. Hence

$$
\begin{aligned}
E(f ; h) & =f^{(k)}(0)-\frac{1}{h^{k}} \sum_{i=0}^{n} a_{i} \tilde{f}\left(h x_{i}\right) \\
& =\left[f^{(k)}(0)-\frac{1}{h^{k}} \sum_{i=0}^{n} a_{i} f\left(h x_{i}\right)\right]+\frac{1}{h^{k}} \sum_{i=0}^{n} a_{i}\left(f\left(h x_{i}\right)-\tilde{f}\left(h x_{i}\right)\right) \\
& =\frac{1}{h^{k}}\left[R_{D^{(k)}}(f ; h)+S(f ; h)\right],
\end{aligned}
$$

so

$$
|E(f ; h)| \leq \frac{1}{h^{k}}\left[\left|R_{D^{(k)}}(f ; h)\right|+|S(f ; h)|\right] .
$$

For the truncation error

$$
\left|R_{D^{(k)}}(f ; h)\right| \leq h^{l+1-\frac{1}{p}} C_{k ; l, p}\left\|f^{(l+1)}\right\|_{p, I_{h}},
$$

and for the roundoff error we have

$$
S(f ; h)=\sum_{i=0}^{n} a_{i}\left(f\left(h x_{i}\right)-\tilde{f}\left(h x_{i}\right)\right)=\sum_{i=0}^{n} a_{i} \mathrm{e}_{i} .
$$

So

$$
|S(f ; h)|=\left|\sum_{i=0}^{n} a_{i} \mathrm{e}_{i}\right| \leq \sum_{i=0}^{n}\left|a_{i}\right|\left|\varepsilon_{i}\right| \leq\|\vec{a}\|_{q}\|\overrightarrow{\mathrm{e}}\|_{p},
$$

where $\|\vec{a}\|_{q}$ is independant of $h$.

Consequently we have

$$
\left|E_{n}(f ; h)\right| \leq h^{l+1-\frac{1}{p}-k} C_{k ; l, p}\left\|f^{(l+1)}\right\|_{p, I_{h}}+\frac{\|\vec{a}\|_{q}\|\overrightarrow{\mathrm{e}}\|_{p}}{h^{k}},
$$

for $l=k, \ldots, k_{a}$. This expression shows that the derivation process $(k>0)$ is numerically unstable. See also [14] for more precision on stability of such processes.

\section{Corrected numerical differentiation rules}

\subsection{The idea}

In this section we suggest a way to improve the order of the truncation error term when we have a primitive $F(x)$ of $f(x)$, which means that $F^{\prime}(x)=f(x)$. Suppose that

$$
h^{k} f^{(k)}(0)=\sum_{i=0}^{n} a_{i} f\left(h x_{i}\right)+o\left(h^{k_{a}}\right),
$$

so the degree of accuracy of the process is $k_{a}$, and the truncation error is

$$
R_{D^{(k)}}(f ; h)=h^{k} f^{(k)}(0)-\sum_{i=0}^{n} a_{i} f\left(h x_{i}\right)=o\left(h^{k_{a}}\right) .
$$


Suppose now that using the same $(n+1)$-dimensional vector of distinct coordinates (or nodes) $\vec{x}=\left(x_{0}, \ldots, x_{n}\right)$ we can find a $(n+1)$-dimensional weight vector $\vec{b}=\left(b_{0}, \ldots, b_{n}\right)$ such that we can determine an expression of the form

$$
\frac{1}{h} \sum_{j=0}^{m} \beta_{j} F\left(h \xi_{j}\right)=\sum_{i=0}^{n} b_{i} f\left(h x_{i}\right)+o\left(h^{k_{a}}\right),
$$

for two $(m+1)$-dimensional vectors $\vec{\beta}=\left(\beta_{0}, \ldots, \beta_{m}\right)$ and $\vec{\xi}=\left(\xi_{0}, \ldots, \xi_{m}\right)$. Its truncation error is

$$
R_{P}(f ; h)=\frac{1}{h} \sum_{j=0}^{m} \beta_{j} F\left(h \xi_{j}\right)-\sum_{i=0}^{n} b_{i} f\left(h x_{i}\right)
$$

and

$$
R_{P}(f ; h)=o\left(h^{k_{a}}\right) .
$$

We can combine the two truncation error terms as follows

$$
R_{D^{(k)}}^{c}(f ; h)=R_{D^{(k)}}(f ; h)-c R_{P}(f ; h)
$$

to get (1.3), and this expression is at least of degree of accuracy $k_{a}$. Since the error terms are both $o\left(h^{k_{a}}\right)$, this expression is also exact for polynomials of degree $\leq k_{a}$. We can select the parameter c such that $R_{D^{(k)}}^{c}(f ; h)$ will be also exact for polynomials of degree $k_{a}+1$. Indeed, if

$$
c=\frac{R_{D^{(k)}}\left(x^{k_{a}+1} ; h\right)}{R_{P}\left(x^{k_{a}+1} ; h\right)},
$$

then $R_{D^{(k)}}^{c}(f ; h)$ will be also exact for polynomials of degree $k_{a}+1$, so its degree of accuracy will be at least $k_{a}+1$.

We will have

$$
\begin{aligned}
h^{k} f^{(k)}(0) & =\sum_{i=0}^{n} a_{i} f\left(h x_{i}\right)+c\left[\frac{1}{h} \sum_{j=0}^{m} \beta_{j} F\left(h \xi_{j}\right)-\sum_{i=0}^{n} b_{i} f\left(h x_{i}\right)\right]+o\left(h^{k_{a}+1}\right) \\
& =\sum_{i=0}^{n}\left[a_{i}-c b_{i}\right] f\left(h x_{i}\right)+\frac{c}{h} \sum_{j=0}^{m} \beta_{j} F\left(h \xi_{j}\right)+o\left(h^{k_{a}+1}\right),
\end{aligned}
$$

or

$$
f^{(k)}(0)=\frac{1}{h^{k}} \sum_{i=0}^{n}\left[a_{i}-c b_{i}\right] f\left(h x_{i}\right)+\frac{c}{h^{k+1}} \sum_{j=0}^{m} \beta_{j} F\left(h \xi_{j}\right)+o\left(h^{k_{a}+1-k}\right),
$$

which will be exact for polynomials of degree up to $k_{a}+1$, and we have increased the order of the error term.

\subsection{Existence}

The vectors $\vec{b}, \vec{\beta}$, and $\vec{\xi}$ of

$$
\frac{1}{h} \sum_{j=0}^{m} \beta_{j} F\left(h \xi_{j}\right)=\sum_{i=0}^{n} b_{i} f\left(h x_{i}\right)
$$

can be determined using the method of undetermined coefficients. It is required that

$$
\sum_{j=0}^{m} \beta_{j}=0
$$

and

$$
\sum_{j=0}^{m} \beta_{j} \frac{\xi_{j}^{l+1}}{l+1}=\sum_{i=0}^{n} b_{i} x_{i}^{l}
$$

for $l=0, \ldots, n$. We also need that

$$
\sum_{j=0}^{m} \beta_{j} \frac{\xi_{j}^{l+1}}{l+1}=\sum_{i=0}^{n} b_{i} x_{i}^{l} .
$$

for $l=n+1, \ldots, k_{a}$. We will not present a complete analysis of this problem here. Examples of solutions of these equations are given in the last section of this paper. 


\subsection{Total error}

In effective numerical computation, with these corrected rules the quantity $f^{(k)}(0)$ is approximated by

$$
f^{(k)}(0) \approx \frac{1}{h^{k}} \sum_{i=0}^{n}\left[a_{i}-c b_{i}\right] \tilde{f}\left(h x_{i}\right)+\frac{c}{h^{k+1}} \sum_{j=0}^{m} \beta_{j} \tilde{F}\left(h \xi_{j}\right),
$$

which uses $\tilde{f}\left(h x_{i}\right)$ and $\tilde{F}\left(h \xi_{j}\right)$ instead of $f\left(h x_{i}\right)$ and $F\left(\xi_{j}\right)$. So roundoff errors are introduced as $e_{i}=f\left(h x_{i}\right)-\tilde{f}\left(h x_{i}\right)$ and $E_{j}=f\left(h x_{j}\right)-\tilde{f}\left(h x_{j}\right)$. The total error $E^{c}(f ; h)$ is decomposed in two types of error: the truncation error $R_{D^{(k)}}^{c}(f ; h)$ and the roundoff error $S^{c}(f ; h)$. Hence

$$
E^{c}(f ; h)=\frac{1}{h^{k}}\left[R_{D^{(k)}}^{c}(f ; h)+S^{c}(f ; h)\right],
$$

where

$$
R_{D^{(k)}}^{c}(f ; h)=h^{k} f^{(k)}(0)-\sum_{i=0}^{n}\left[a_{i}-c b_{i}\right] f\left(h x_{i}\right)+\frac{c}{h^{1}} \sum_{j=0}^{m} \beta_{j} F\left(h \xi_{j}\right),
$$

and

$$
S^{c}(f ; h)=\sum_{i=0}^{n}\left[a_{i}-c b_{i}\right]\left(f\left(h x_{i}\right)-\tilde{f}\left(h x_{i}\right)\right)+\frac{c}{h^{1}} \sum_{j=0}^{m} \beta_{j}\left(F\left(h \xi_{j}\right)-\tilde{F}\left(h \xi_{j}\right)\right) .
$$

For the truncation error, if we proceed as we did in the preceding section, we could establish the bound

$$
\left|R_{D^{(k)}}^{c}(f ; h)\right| \leq h^{l+1-\frac{1}{p}} C_{k ; l, p}^{c}\left\|f^{(l+1)}\right\|_{p, I_{h}} .
$$

For the roundoff error we have

$$
\begin{aligned}
S^{c}(f ; h) & =\sum_{i=0}^{n}\left[a_{i}-c b_{i}\right]\left(f\left(h x_{i}\right)-\tilde{f}\left(h x_{i}\right)\right)+\frac{c}{h} \sum_{j=0}^{m} \beta_{j}\left(F\left(h \xi_{j}\right)-\tilde{F}\left(\xi_{j}\right)\right) \\
& =\sum_{i=0}^{n}\left[a_{i}-c b_{i}\right] \mathrm{e}_{i}+\frac{c}{h} \sum_{j=0}^{m} \beta_{j} E_{i},
\end{aligned}
$$

so

$$
\begin{aligned}
\left|S^{c}(f ; h)\right| & \leq \sum_{i=0}^{n}\left|a_{i}-c b_{i}\right|\left|e_{i}\right|+\frac{c}{h} \sum_{j=0}^{m}\left|\beta_{j}\right|\left|E_{i}\right| \\
& \leq\|\vec{a}-c \vec{b}\|_{q}\|\vec{e}\|_{p}+\frac{c}{h}\|\vec{\beta}\|_{q}\|\vec{E}\|_{p}
\end{aligned}
$$

where $\|\vec{a}-c \vec{b}\|_{q}$ and $\|\vec{\beta}\|_{q}$ are independant of $h$.

Consequently we have

$$
\left|E^{c}(f ; h)\right| \leq h^{l+1-\frac{1}{p}-k} C_{k ; l, p}^{c}\left\|f^{(l+1)}\right\|_{p, I_{h}}+\frac{1}{h^{k}}\left[\|\vec{a}-c \vec{b}\|_{q}\|\overrightarrow{\mathrm{e}}\|_{p}+\frac{c}{h}\|\vec{\beta}\|_{q}\|\vec{E}\|_{p}\right],
$$

not only for $l=k, \ldots, k_{a}$ but also for $l=k_{a}+1$. Obviously, this process is intereating for regular functions $f(x) \in A C^{k_{a}+2, p}\left(I_{h}\right)$ for which we know a primitive.

\section{Examples of formula}

Example 5.1. First derivative: the 2-points symmetric formula is

$$
f^{(1)}(0)=\frac{1}{2 h}[f(h)-f(-h)]+o(h),
$$


which is exact for polynomials of degree up to 2. The truncation error we consider is

$$
R_{D^{(1)}}(f ; h)=h f^{(1)}(0)-\frac{1}{2}[f(h)-f(-h)]=o\left(h^{2}\right) .
$$

For $f(x)=x^{l}, R_{D^{(1)}}\left(x^{l} ; h\right)=0$ for $l=0,1,2$, and for $l \geq 3$ we have

$$
R_{D^{(1)}}\left(x^{l} ; h\right)=-h^{l} \frac{\left[1-(-1)^{l}\right]}{2}=\left\{\begin{array}{lll}
0 & \text { for even } l, \\
-h^{l} & \text { for odd } & l .
\end{array}\right.
$$

The corresponding expression involving the primitive $F(x)$ is

$$
\frac{1}{h}[F(h)-2 F(0)+F(-h)]=\frac{1}{2}[f(h)-f(-h)]+o\left(h^{2}\right) .
$$

with its truncation error

$$
R_{P}(f ; h)=\frac{1}{h}[F(h)-2 F(0)+F(-h)]-\frac{1}{2}[f(h)-f(-h)]=o\left(h^{2}\right) .
$$

For $f(x)=x^{l}, R_{P}\left(x^{l} ; h\right)=0$ for $l=0,1,2$, and for $l \geq 3$ we have

$$
R_{P}\left(x^{l} ; h\right)=h^{l}\left[1-(-1)^{l}\right]\left[\frac{1}{l+1}-\frac{1}{2}\right]= \begin{cases}0 & \text { for even } l \\ 2 h^{l}\left[\frac{1}{l+1}-\frac{1}{2}\right] & \text { for odd }\end{cases}
$$

Then we choose

$$
c=\frac{R_{D^{(1)}}\left(x^{3} ; h\right)}{R_{P}\left(x^{3} ; h\right)}=\frac{R_{D^{(1)}}\left(x^{3} ; 1\right)}{R_{P}\left(x^{3} ; 1\right)}=\frac{-1}{-1 / 2}=2 .
$$

The resulting formula will be exact not only for polynomials of degree 3 but also for polynomial of degree 4 , since (5.1) and (5.2) hold. We obtain

$$
h f^{(1)}(0)=-\frac{1}{2}[f(h)-f(-h)]+\frac{2}{h}[F(h)-2 F(0)+F(-h)]+o\left(h^{4}\right)
$$

or

$$
f^{(1)}(0)=-\frac{1}{2 h}[f(h)-f(-h)]+\frac{2}{h^{2}}[F(h)-2 F(0)+F(-h)]+o\left(h^{3}\right) .
$$

Example 5.2. First derivative: the one-sided formula is

$$
f^{(1)}(0)=\frac{1}{h}[f(h)-f(0)]+o(1) .
$$

Its corresponding truncation error is

$$
R_{D^{(1)}}(f ; h)=h f^{(1)}(0)-[f(h)-f(0)]=o(h) .
$$

For $f(x)=x^{l}, R_{D^{(1)}}\left(x^{l} ; h\right)=0$ for $l=0,1$, and for $l \geq 2$ we have

$$
R_{D^{(1)}}\left(x^{l} ; h\right)=-h^{l}
$$

We consider

$$
\frac{1}{h}[F(h)-F(0)]=\frac{1}{2}[f(h)+f(0)]+o(h) .
$$

with its truncation error

$$
R_{P}(f ; h)=\frac{1}{h}[F(h)-F(0)]-\frac{1}{2}[f(h)+f(0)]=o(h) .
$$


For $f(x)=x^{l}, R_{P}\left(x^{l} ; h\right)=0$ for $l=0,1$, and for $l \geq 2$ we have

$$
R_{P}\left(x^{l} ; h\right)=h^{l}\left[\frac{1}{l+1}-\frac{1}{2}\right] \text {. }
$$

Then we choose

$$
c=\frac{R_{D^{(1)}}\left(x^{2} ; h\right)}{R_{P}\left(x^{2} ; h\right)}=\frac{R_{D^{(1)}}\left(x^{2} ; 1\right)}{R_{P}\left(x^{2} ; 1\right)}=\frac{-1}{-1 / 6}=6 .
$$

The resulting formula will be exact for polynomial of degree 2, and we obtain

$$
h f^{(1)}(0)=-2[f(h)+2 f(0)]+\frac{6}{h}[F(h)-F(0)]+o\left(h^{2}\right)
$$

or

$$
f^{(1)}(0)=-\frac{2}{h}[f(h)+2 f(0)]+\frac{2}{h^{2}}[F(h)-F(0)]+o(h) .
$$

Example 5.3. Second derivative: the 3-points symmetric formula is

$$
f^{(2)}(0)=\frac{1}{h^{2}}[f(h)-2 f(0)+f(-h)]+o(h),
$$

and its truncation error

$$
R_{D^{(2)}}(f ; h)=h^{2} f^{(2)}(0)-[f(h)-2 f(0)+f(-h)]=o\left(h^{3}\right) .
$$

For $f(x)=x^{l}, R_{D^{(2)}}\left(x^{l} ; h\right)=0$ for $l=0,1,2,3$, and for $l \geq 4$ we have

$$
R_{D^{(2)}}\left(x^{l} ; h\right)=-h^{l}\left[1+(-1)^{l}\right]=\left\{\begin{array}{lll}
0 & \text { for odd } l & l \\
-2 h^{l} & \text { for even } & l .
\end{array}\right.
$$

We consider

$$
\frac{1}{h}[F(h)-F(-h)]=\left[\frac{1}{3} f(h)+\frac{4}{3} f(0)+\frac{1}{3} f(-h)\right]+o\left(h^{3}\right),
$$

with its truncation error

$$
R_{P}(f ; h)=\frac{1}{h}[F(h)-F(-h)]-\left[\frac{1}{3} f(h)+\frac{4}{3} f(0)+\frac{1}{3} f(-h)\right]=o\left(h^{3}\right) .
$$

For $f(x)=x^{l}, R_{P}\left(x^{l} ; h\right)=0$ for $l=0,1,2,3$, and for $l \geq 4$ we have

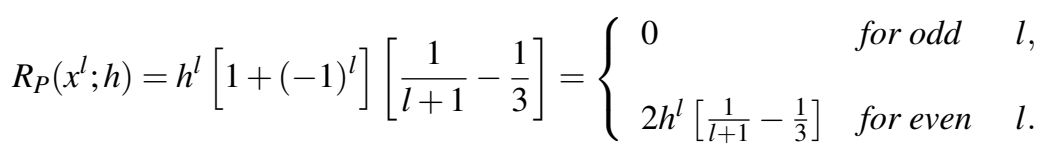

Then we choose

$$
c=\frac{R_{D^{(2)}}\left(x^{4} ; h\right)}{R_{P}\left(x^{4} ; h\right)}=\frac{R_{D^{(2)}}\left(x^{4} ; 1\right)}{R_{P}\left(x^{4} ; 1\right)}=\frac{-2}{-4 / 15}=\frac{15}{2} .
$$

The resulting formula is not only exact for polynomials of degree 4 but also for polynomials of degree 5, since (5.3) and (5.4) hold. We obtain

$$
h^{2} f^{(2)}(0)=-\frac{3}{2}[f(h)+8 f(0)+f(-h)]+\frac{15}{2 h}[F(h)-F(-h)]+o\left(h^{5}\right)
$$

or

$$
f^{(2)}(0)=-\frac{3}{2 h^{2}}[f(h)+8 f(0)+f(-h)]+\frac{15}{2 h^{3}}[F(h)-F(-h)]+o\left(h^{3}\right) .
$$


Example 5.4. Second derivative: the 4-points symmetric formula is

$$
f^{(2)}(0)=\frac{9}{8 h^{2}}\left[f(h)-f\left(\frac{h}{3}\right)-f\left(-\frac{h}{3}\right)+f(-h)\right]+o(h) .
$$

Its truncation error is

$$
R_{D^{(2)}}(f ; h)=h^{2} f^{(2)}(0)-\frac{9}{8}\left[f(h)-f\left(\frac{h}{3}\right)-f\left(-\frac{h}{3}\right)+f(-h)\right]=o\left(h^{3}\right) .
$$

For $f(x)=x^{l}, R_{D^{(2)}}\left(x^{l} ; h\right)=0$ for $l=0,1,2,3$, and for $l \geq 4$ we have

$$
R_{D^{(2)}}\left(x^{l} ; h\right)=-\frac{9}{8} h^{l}\left[1+(-1)^{l}\right]\left[1-\frac{1}{3^{l}}\right]=\left\{\begin{array}{lll}
0 & \text { for odd } l, \\
-\frac{9}{4} h^{l}\left[1-\frac{1}{3^{l-1}}\right] & \text { for even } l .
\end{array}\right.
$$

We consider

$$
\frac{1}{h}[F(h)-F(-h)]=\frac{1}{4}\left[f(h)+3 f\left(\frac{h}{3}\right)+3 f\left(-\frac{h}{3}\right)+f(-h)\right]+o\left(h^{3}\right) .
$$

with its truncation error

$$
R_{P}(f ; h)=\frac{1}{h}[F(h)-F(-h)]-\frac{1}{4}\left[f(h)+3 f\left(\frac{h}{3}\right)+3 f\left(-\frac{h}{3}\right)+f(-h)\right]=o\left(h^{3}\right) .
$$

For $f(x)=x^{l}, R_{P}\left(x^{l} ; h\right)=0$ for $l=0,1,2,3$, and for $l \geq 4$ we have

$$
R_{P}\left(x^{l} ; h\right)=h^{l}\left[1+(-1)^{l}\right]\left[\frac{1}{l+1}-\frac{1}{4}\left(1+\frac{1}{3^{l-1}}\right)\right]= \begin{cases}0 & \text { for odd } l, \\ 2 h^{l}\left[\frac{1}{l+1}-\frac{1}{4}\left(1+\frac{1}{3^{l-1}}\right)\right] & \text { for even } l .\end{cases}
$$

Then we choose

$$
c=\frac{R_{D^{(2)}}\left(x^{4} ; h\right)}{R_{P}\left(x^{4} ; h\right)}=\frac{R_{D^{(2)}}\left(x^{4} ; 1\right)}{R_{P}\left(x^{4} ; 1\right)}=\frac{-20 / 9}{-16 / 135}=\frac{75}{4} .
$$

The resulting formula will be exact not only for polynomials of degree 4 but also for polynomials of degree 5 since (5.5) and (5.6) hold, and we obtain

$$
h^{2} f^{(2)}(0)=-\frac{1}{16}\left[57 f(h)+243 f\left(\frac{h}{3}\right)+243 f\left(-\frac{h}{3}\right)+57 f(-h)\right]+\frac{75}{4 h}[F(h)-F(-h)]+o\left(h^{5}\right)
$$

or

$$
f^{(2)}(0)=-\frac{1}{16 h^{2}}\left[57 f(h)+243 f\left(\frac{h}{3}\right)+243 f\left(-\frac{h}{3}\right)+57 f(-h)\right]+\frac{75}{4 h^{3}}[F(h)-F(-h)]+o\left(h^{3}\right) .
$$

Example 5.5. Third derivative: the 4-point symmetric formula is

$$
f^{(3)}(0)=\frac{27}{8 h^{3}}\left[f(h)-3 f\left(\frac{h}{3}\right)+3 f\left(-\frac{h}{3}\right)-f(-h)\right]+o(h) .
$$

Its truncation error is

$$
R_{D^{(3)}}(f ; h)=h^{3} f^{(3)}(0)-\frac{27}{8}\left[f(h)-3 f\left(\frac{h}{3}\right)+3 f\left(-\frac{h}{3}\right)-f(-h)\right]=o\left(h^{4}\right) .
$$

For $f(x)=x^{l}, R_{D^{(3)}}\left(x^{l} ; h\right)=0$ for $l=0,1,2,3,4$, and for $l \geq 5$ we have

$$
R_{D^{(3)}}\left(x^{l} ; h\right)=-\frac{27}{8} h^{l}\left[1-(-1)^{l}\right]\left[1-\frac{1}{3^{l-1}}\right]=\left\{\begin{array}{lll}
0 & \text { for even } & l, \\
-\frac{27}{4} h^{l}\left[1-\frac{1}{3^{l-1}}\right] & \text { for odd } & l .
\end{array}\right.
$$


We consider

$$
\frac{1}{h}[F(-h)-2 F(0)+F(h)]=\frac{1}{32}\left[7 f(h)+27 f\left(\frac{h}{3}\right)-27 f\left(-\frac{h}{3}\right)-7 f(-h)\right]+o\left(h^{4}\right) .
$$

with its truncation error

$$
R_{P}(f ; h)=\frac{1}{h}[F(h)-2 F(0)-F(-h)]-\frac{1}{32}\left[7 f(h)+27 f\left(\frac{h}{3}\right)-27 f\left(-\frac{h}{3}\right)-7 f(-h)\right]=o\left(h^{4}\right) .
$$

For $f(x)=x^{l}, R_{P}\left(x^{l} ; h\right)=0$ for $l=0,1,2,3,4$, and for $l \geq 5$ we have

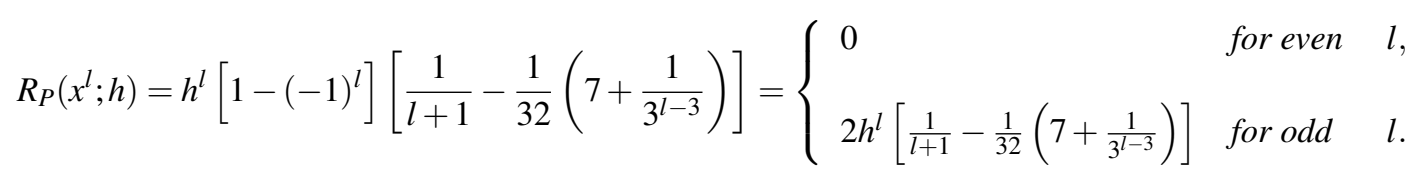

Then we choose

$$
c=\frac{R_{D^{(3)}}\left(x^{5} ; h\right)}{R_{P}\left(x^{5} ; h\right)}=\frac{R_{D^{(3)}}\left(x^{5} ; 1\right)}{R_{3}^{P}\left(x^{5} ; 1\right)}=\frac{-20 / 3}{-1 / 9}=60 .
$$

The resulting formula will also be exact for polynomials of degree 4 since (5.7) and (5.8) hold, and we obtain

$$
h^{3} f^{(3)}(0)=-\frac{1}{4}\left[39 f(h)+243 f\left(\frac{h}{3}\right)-243 f\left(-\frac{h}{3}\right)-39 f(-h)\right]+\frac{60}{h}[F(h)-2 F(0)+F(-h)]+o\left(h^{6}\right)
$$

or

$$
f^{(3)}(0)=-\frac{1}{4 h^{3}}\left[39 f(h)+243 f\left(\frac{h}{3}\right)-243 f\left(-\frac{h}{3}\right)-39 f(-h)\right]+\frac{60}{h^{4}}[F(h)-2 F(0)+F(-h)]+o\left(h^{3}\right) .
$$

Example 5.6. Fourth derivative: the 5-points symmetric formula is

$$
f^{(4)}(0)=\frac{16}{h^{4}}\left[f(h)-4 f\left(\frac{h}{2}\right)+6 f(0)-4 f\left(-\frac{h}{2}\right)+f(-h)\right]+o(h) .
$$

Its truncation error is

$$
R_{D^{(4)}}(f ; h)=h^{4} f^{(4)}(0)-16\left[f(h)-4 f\left(\frac{h}{2}\right)+6 f(0)-4 f\left(-\frac{h}{2}\right)+f(-h)\right]=o\left(h^{5}\right) .
$$

For $f(x)=x^{l}, R_{D^{(4)}}\left(x^{l} ; h\right)=0$ for $l=0,1,2,3,4,5$, and for $l \geq 6$ we have

$$
R_{D^{(4)}}\left(x^{l} ; h\right)=-16 h^{l}\left[1+(-1)^{l}\right]\left[1-\frac{1}{2^{l-2}}\right]=\left\{\begin{array}{lll}
0 & \text { for odd } & l, \\
-32 h^{l}\left[1-\frac{1}{2^{l-2}}\right] & \text { for even } & l .
\end{array}\right.
$$

We consider

$$
\frac{1}{h}[F(h)-F(-h)]=\frac{1}{45}\left[7 f(h)+32 f\left(\frac{h}{2}\right)+12 f(0)+32 f\left(-\frac{h}{2}\right)+7 f(-h)\right]+o\left(h^{5}\right) .
$$

with its truncation error

$$
R_{P}(f ; h)=\frac{1}{h}[F(h)-F(-h)]-\frac{1}{45}\left[7 f(h)+32 f\left(\frac{h}{2}\right)+12 f(0)+32 f\left(-\frac{h}{2}\right)+7 f(-h)\right]=o\left(h^{5}\right) .
$$

For $f(x)=x^{l}, R_{P}\left(x^{l} ; h\right)=0$ for $l=0,1,2,3,4,5$, and for $l \geq 6$ we have

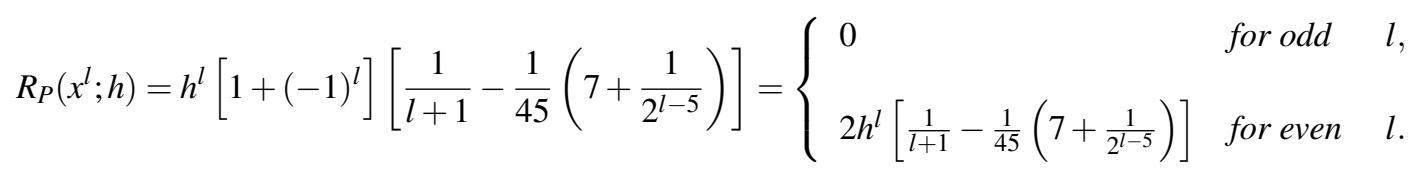


Then we choose

$$
c=\frac{R_{D^{(4)}}\left(x^{6} ; h\right)}{R_{P}\left(x^{6} ; h\right)}=\frac{R_{D^{(4)}}\left(x^{6} ; 1\right)}{R_{P}\left(x^{6} ; 1\right)}=\frac{-30}{-1 / 21}=630 .
$$

The resulting formula will also be exact not only for polynomials of degree 6 but also for polynomials of degree 7 since (5.9) and (5.10) hold. We obtain

$$
h^{4} f^{(4)}(0)=-\left[82 f(h)+512 f\left(\frac{h}{2}\right)+72 f(0)+512 f\left(-\frac{h}{2}\right)+82 f(-h)\right]+\frac{630}{h}[F(h)-F(-h)]+o\left(h^{7}\right)
$$

or

$$
f^{(4)}(0)=-\frac{1}{h^{4}}\left[82 f(h)+512 f\left(\frac{h}{2}\right)+72 f(0)+512 f\left(-\frac{h}{2}\right)+82 f(-h)\right]+\frac{630}{h^{5}}[F(h)-F(-h)]+o\left(h^{3}\right)
$$

\section{Numerical examples}

To illustrate the results, we will apply the formulae to the functions given in Table 1. To get the exponent $L$ of expression of the form $o\left(h^{L}\right)$, we compute the absolute error which is of the form $O\left(h^{L+1}\right)$ for a regular enough function, which is the case for the chosen functions in Table 1. In the formula, we will replace $o\left(h^{L}\right)$ by $O\left(h^{L+1}\right)$. So the order $L+1$ is estimated by the expression

$$
\log _{2}\left(\frac{\text { absolute error for } h}{\text { absolute error for } h / 2}\right) \approx \log _{2}\left(\frac{O\left(h^{L+1}\right)}{O\left((h / 2)^{L+1}\right)}\right) \approx \log _{2}\left(2^{L+1}\right)=L+1
$$

The approximations of $L+1$ are given in the last column of the tables below. Obviously, the derivative can be estimated at any value $a$ not only at 0 as expressed in the formula. We reconsider the 6 examples of the preceding section, and we numerically observe the predicted order $L+1$ of $O\left(h^{L+1}\right)$.

Example 6.1. First derivative: the 2-points symmetric formula is

$$
f^{(1)}(0)=\frac{1}{2 h}[f(h)-f(-h)]+O\left(h^{2}\right),
$$

and the corrected formula is

$$
f^{(1)}(0)=-\frac{1}{2 h}[f(h)-f(-h)]+\frac{2}{h^{2}}[F(h)-2 F(0)+F(-h)]+O\left(h^{4}\right) .
$$

The numerical results are given in Table 2, which indicates the order of the method.

Example 6.2. First derivative: the one-sided formula is

$$
f^{(1)}(0)=\frac{1}{h}[f(h)-f(0)]+O(h),
$$

and the corrected formula is

$$
f^{(1)}(0)=-\frac{2}{h}[f(h)+2 f(0)]+\frac{2}{h^{2}}[F(h)-F(0)]+O\left(h^{2}\right) .
$$

The numerical results are given in Table 3, which indicates the order of the method.

Example 6.3. Second derivative: the 3-points symmetric formula is

$$
f^{(2)}(0)=\frac{1}{h^{2}}[f(h)-2 f(0)+f(-h)]+O\left(h^{2}\right),
$$

and the corrected formula is

$$
f^{(2)}(0)=-\frac{3}{2 h^{2}}[f(h)+8 f(0)+f(-h)]+\frac{15}{2 h^{3}}[F(h)-F(-h)]+O\left(h^{4}\right) .
$$

The numerical results are given in Table 4, which indicates the order of the method. 
Example 6.4. Second derivative: the 4-points symmetric formula is

$$
f^{(2)}(0)=\frac{9}{8 h^{2}}\left[f(h)-f\left(\frac{h}{3}\right)-f\left(-\frac{h}{3}\right)+f(-h)\right]+O\left(h^{2}\right) .
$$

and

$$
f^{(2)}(0)=-\frac{1}{16 h^{2}}\left[57 f(h)+243 f\left(\frac{h}{3}\right)+243 f\left(-\frac{h}{3}\right)+57 f(-h)\right]+\frac{75}{4 h^{3}}[F(h)-F(-h)]+O\left(h^{4}\right) .
$$

The numerical results are given in Table 5, which indicates the order of the method.

Example 6.5. Third derivative: the 4-point symmetric formula is

$$
f^{(3)}(0)=\frac{27}{8 h^{3}}\left[f(h)-3 f\left(\frac{h}{3}\right)+3 f\left(-\frac{h}{3}\right)-f(-h)\right]+O\left(h^{2}\right) .
$$

and

$$
f^{(3)}(0)=-\frac{1}{4 h^{3}}\left[39 f(h)+243 f\left(\frac{h}{3}\right)-243 f\left(-\frac{h}{3}\right)-39 f(-h)\right]+\frac{60}{h^{4}}[F(h)-2 F(0)+F(-h)]+O\left(h^{4}\right) .
$$

The numerical results are given in Table 6, which indicates the order of the method.

Example 6.6. Fourth derivative: the 5-points symmetric formula is

$$
f^{(4)}(0)=\frac{16}{h^{4}}\left[f(h)-4 f\left(\frac{h}{2}\right)+6 f(0)-4 f\left(-\frac{h}{2}\right)+f(-h)\right]+O\left(h^{2}\right) .
$$

and

$$
f^{(4)}(0)=-\frac{1}{h^{4}}\left[82 f(h)+512 f\left(\frac{h}{2}\right)+72 f(0)+512 f\left(-\frac{h}{2}\right)+82 f(-h)\right]+\frac{630}{h^{5}}[F(h)-F(-h)]+O\left(h^{4}\right)
$$

The numerical results are given in Table 7, which indicates the order of the method.

\section{Conclusion}

In this paper, we have presented a complete analysis of the standard numerical differentiation formulae for which we have established, using two different methods, the best error bounds depending on the regularity of absolutely continuous functions. Moreover we have presented a way to improve the order of those formulae by adding information coming from a primitive of the function. Obviously, this process is possible if we can get values of the primitive, directly of by an indirect method.

\section{Acknowledgement}

This work has been financially supported by an individual discovery grant from NSERC (Natural Sciences and Engineering Research Council of Canada).

\section{References}

[1] P. J. Davis, Interpolation and Approximation, Dover, N.Y., 1975.

[2] A. S. Househoulder, Principles of Numerical Analysis, McGraw Hill, Columbus, N.Y., 1953.

[3] D. Kincaid, W. Cheney, Numerical Analysis, Brooks/Cole Pub. Co., Cal., 1991.

[4] A. Ralston, A First Course in Numerical Analysis, McGraw-Hill, N.Y., 1965.

[5] N. Macon, A. Spitzbart, Inverses of Vandermonde matrices, Amer. Math. Monthly, 65(2) (1958), 95-100. http://dx.doi.org/10.2307/2308881

[6] E. Asplund, L. Bungart, A First Course in Integration, Holt, Rinehart and Winston, N.Y., 1966.

[7] L. L. Schumaker, Spline Functions Basic Theory, Wiley, N.Y., 1981. 


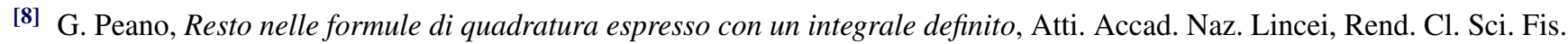
Mat. Nat., Serie 5, 22(I) (1913), 562-569.

[9] R. von Mises, Über allgemeine quadraturformeln, J. Reine Angew. Math., 174 (1935), 56-67; reprinted in Selected Papers of Richard von Mises, Vol. 1, 559-574, American Mathematical Society, Providence, R.I., 1963.

[10] A. Ghizzetti, A. Ossicini, Quadrature Formulae, Academic Press, N.Y., 1970.

${ }^{[11]}$ F. Dubeau, Revisited optimal error bounds for interpolatory integration rules, Adv. Numer. Anal., 2016 (2016), Article ID 3170595, 8 pages, http://dx.doi.org/10.1155/2016/3170595.

${ }^{[12]}$ F. Dubeau, The method of undetermined coefficients: general approach and optimal error bounds, J. Math. Anal., 5(4) (2014), 1-11.

[13] J. S. C. Prentice, Truncation and roundoff errors in three-point approximations of first and second derivatives, Appl. Math. Comput., 217 (2011), 4576-4581. http://dx.doi.org/10.1016/j.amc.2010.11.008

[14] N. J. Higham, Accuracy and Stability of Numerical Algorithms, 2nd Ed., SIAM, Philadelphia, PN, 2002. http://dx.doi.org/10.1137/1.9780898718027

\begin{tabular}{|c|c|c|}
\hline \multicolumn{3}{|c|}{ Test functions } \\
\hline$f(x)$ & $\frac{1}{1+x^{2}}$ & $\tan (x)$ \\
\hline$f^{(1)}(x)$ & $-2 \frac{x}{\left(1+x^{2}\right)^{2}}$ & $1+\tan ^{2}(x)$ \\
\hline$f^{(2)}(x)$ & $-2 \frac{\left(1-3 x^{2}\right)}{\left(1+x^{2}\right)^{3}}$ & $2 \tan (x)\left(1+\tan ^{2}(x)\right)$ \\
\hline$f^{(3)}(x)$ & $24 x \frac{\left(1-x^{2}\right)}{\left(1+x^{2}\right)^{4}}$ & $2\left(1+\tan ^{2}(x)\right)\left(1+3 \tan ^{2}(x)\right)$ \\
\hline$f^{(4)}(x)$ & $24 \frac{\left(1-10 x^{2}+5 x^{4}\right)}{\left(1+x^{2}\right)^{5}}$ & $8 \tan (x)\left(1+\tan ^{2}(x)\right)\left(2+3 \tan ^{2}(x)\right)$ \\
\hline$F(x)$ & $\arctan (x)$ & $-\ln |\cos (x)|$ \\
\hline
\end{tabular}

Table 1. Test functions for numerical differentiation. 


\begin{tabular}{|c|c|c|c|c|c|c|}
\hline \multirow[b]{3}{*}{$h$} & \multicolumn{6}{|c|}{ Symmetric first derivative rule } \\
\hline & \multicolumn{3}{|c|}{ Standard rule } & \multicolumn{3}{|c|}{ Corrected rule } \\
\hline & $\begin{array}{l}\text { computed } \\
\text { derivative }\end{array}$ & $\begin{array}{c}\text { absolute } \\
\text { error }\end{array}$ & $\begin{array}{c}\text { estimated } \\
\text { order }\end{array}$ & $\begin{array}{l}\text { computed } \\
\text { derivative }\end{array}$ & $\begin{array}{c}\text { absolute } \\
\text { error }\end{array}$ & $\begin{array}{c}\text { estimated } \\
\text { order }\end{array}$ \\
\hline & \multicolumn{6}{|c|}{ Estimation of $f^{(1)}(2)=-0.16$ for $f(x)=1 /\left(1+x^{2}\right)$} \\
\hline 1.0000 & $-2.00000000 \mathrm{e}-01$ & $4.00000000 \mathrm{e}-02$ & & $-1.59707000 \mathrm{e}-01$ & $2.93000415 \mathrm{e}-04$ & \\
\hline 0.5000 & $-1.69761273 \mathrm{e}-01$ & $9.76127321 \mathrm{e}-03$ & 2.03 & $-1.59948828 \mathrm{e}-01$ & $5.11719430 \mathrm{e}-05$ & 2.52 \\
\hline 0.2500 & $-1.62410785 \mathrm{e}-01$ & $2.41078509 \mathrm{e}-03$ & 2.02 & $-1.59996441 \mathrm{e}-01$ & $3.55871874 \mathrm{e}-06$ & 3.85 \\
\hline 0.1250 & $-1.60600684 \mathrm{e}-01$ & $6.00684200 \mathrm{e}-04$ & 2.00 & $-1.59999772 \mathrm{e}-01$ & $2.27514621 \mathrm{e}-07$ & 3.98 \\
\hline 0.0625 & $-1.60150043 \mathrm{e}-01$ & $1.50042917 \mathrm{e}-04$ & 2.00 & $-1.59999986 \mathrm{e}-01$ & $1.42973008 \mathrm{e}-08$ & 3.99 \\
\hline 0.0313 & $-1.60037503 e-01$ & $3.75026847 \mathrm{e}-05$ & 2.00 & $-1.59999999 \mathrm{e}-01$ & $8.95188673 \mathrm{e}-10$ & 4.00 \\
\hline \multirow[t]{2}{*}{0.0156} & $-1.60009375 \mathrm{e}-01$ & $9.37516783 \mathrm{e}-06$ & 2.00 & $-1.60000000 \mathrm{e}-01$ & $5.67215996 \mathrm{e}-11$ & 3.98 \\
\hline & \multicolumn{6}{|c|}{ Estimation of $f^{(1)}(\pi / 4)=2$ for $f(x)=\tan (x)$} \\
\hline 1.0000 & $-2.18503986 \mathrm{e}+00$ & $4.18503986 \mathrm{e}+00$ & & $3.93847408 \mathrm{e}+00$ & $6.57541629 e+00$ & \\
\hline 0.5000 & $3.11481545 \mathrm{e}+00$ & $1.11481545 \mathrm{e}+00$ & 1.91 & $1.81019631 \mathrm{e}+00$ & $1.89803686 \mathrm{e}-01$ & 5.11 \\
\hline 0.2500 & $2.18520996 \mathrm{e}+00$ & $1.85209959 \mathrm{e}-01$ & 2.59 & $1.99348573 \mathrm{e}+00$ & $6.51426518 \mathrm{e}-03$ & 4.86 \\
\hline 0.1250 & $2.04273537 \mathrm{e}+00$ & $4.27353698 \mathrm{e}-02$ & 2.12 & $1.99963919 \mathrm{e}+00$ & $3.60810092 \mathrm{e}-04$ & 4.17 \\
\hline 0.0625 & $2.01048219 \mathrm{e}+00$ & $1.04821852 \mathrm{e}-02$ & 2.03 & $1.99997809 \mathrm{e}+00$ & $2.19088367 \mathrm{e}-05$ & 4.04 \\
\hline 0.0313 & $2.00260824 \mathrm{e}+00$ & $2.60824212 \mathrm{e}-03$ & 2.01 & $1.99999864 \mathrm{e}+00$ & $1.35955952 \mathrm{e}-06$ & 4.01 \\
\hline 0.0156 & $2.00065130 \mathrm{e}+00$ & $6.51296080 \mathrm{e}-04$ & 2.00 & $1.99999992 \mathrm{e}+00$ & $8.48203887 \mathrm{e}-08$ & 4.00 \\
\hline
\end{tabular}

Table 2. Estimation of $f^{(1)}(x)$ using a symmetric rule.

\begin{tabular}{|c|c|c|c|c|c|c|}
\hline \multirow[b]{3}{*}{$h$} & \multicolumn{6}{|c|}{ Unilateral first derivative rule } \\
\hline & \multicolumn{3}{|c|}{ Standard rule } & \multicolumn{3}{|c|}{ Corrected rule } \\
\hline & $\begin{array}{l}\text { computed } \\
\text { derivative }\end{array}$ & $\begin{array}{c}\text { absolute } \\
\text { error }\end{array}$ & $\begin{array}{c}\text { estimated } \\
\text { order }\end{array}$ & $\begin{array}{l}\text { computed } \\
\text { derivative }\end{array}$ & $\begin{array}{c}\text { absolute } \\
\text { error }\end{array}$ & $\begin{array}{c}\text { estimated } \\
\text { order }\end{array}$ \\
\hline & \multicolumn{6}{|c|}{ Estimation of $f^{(1)}(2)=-0.16$ for $f(x)=1 /\left(1+x^{2}\right)$} \\
\hline 1.0000 & $-1.00000000 \mathrm{e}-01$ & $6.00000000 \mathrm{e}-02$ & & $-1.48617672 \mathrm{e}-01$ & $1.13823276 \mathrm{e}-02$ & \\
\hline 0.5000 & $-1.24137931 \mathrm{e}-01$ & $3.58620690 \mathrm{e}-02$ & 0.74 & $-1.56334573 e-01$ & $3.66542739 \mathrm{e}-03$ & 1.63 \\
\hline 0.2500 & $-1.40206186 \mathrm{e}-01$ & $1.97938144 \mathrm{e}-02$ & 0.86 & $-1.58952804 \mathrm{e}-01$ & $1.04719561 \mathrm{e}-03$ & 1.81 \\
\hline 0.1250 & $-1.49575071 \mathrm{e}-01$ & $1.04249292 \mathrm{e}-02$ & 0.93 & $-1.59719803 e-01$ & $2.80196710 \mathrm{e}-04$ & 1.90 \\
\hline 0.0625 & $-1.54646840 \mathrm{e}-01$ & $5.35315985 \mathrm{e}-03$ & 0.96 & $-1.59927519 \mathrm{e}-01$ & $7.24808122 \mathrm{e}-05$ & 1.95 \\
\hline 0.0313 & $-1.57287102 \mathrm{e}-01$ & $2.71289769 \mathrm{e}-03$ & 0.98 & $-1.59981568 \mathrm{e}-01$ & $1.84323851 \mathrm{e}-05$ & 1.98 \\
\hline \multirow[t]{2}{*}{0.0156} & $-1.58634325 \mathrm{e}-01$ & $1.36567488 \mathrm{e}-03$ & 0.99 & $-1.59995352 \mathrm{e}-01$ & $4.64763034 \mathrm{e}-06$ & 1.99 \\
\hline & \multicolumn{6}{|c|}{ Estimation of $f^{(1)}(\pi / 4)=2$ for $f(x)=\tan (x)$} \\
\hline 1.0000 & $-5.58803782 \mathrm{e}+00$ & $7.58803782 \mathrm{e}+00$ & & $1.23765843 \mathrm{e}+01$ & $2.15169529 \mathrm{e}+01$ & \\
\hline 0.5000 & $4.81644688 \mathrm{e}+00$ & $2.81644688 \mathrm{e}+00$ & 1.43 & $4.68917926 \mathrm{e}-01$ & $1.53108207 \mathrm{e}+00$ & 3.81 \\
\hline 0.2500 & $2.74318567 \mathrm{e}+00$ & $7.43185669 \mathrm{e}-01$ & 1.92 & $1.84910116 \mathrm{e}+00$ & $1.50898839 \mathrm{e}-01$ & 3.34 \\
\hline 0.1250 & $2.29941556 \mathrm{e}+00$ & $2.99415562 \mathrm{e}-01$ & 1.31 & $1.97268790 \mathrm{e}+00$ & $2.73120951 \mathrm{e}-02$ & 2.47 \\
\hline 0.0625 & $2.13630119 \mathrm{e}+00$ & $1.36301191 \mathrm{e}-01$ & 1.14 & $1.99406905 \mathrm{e}+00$ & $5.93095126 \mathrm{e}-03$ & 2.20 \\
\hline 0.0313 & $2.06521013 \mathrm{e}+00$ & $6.52101292 \mathrm{e}-02$ & 1.06 & $1.99861227 \mathrm{e}+00$ & $1.38772563 \mathrm{e}-03$ & 2.10 \\
\hline 0.0156 & $2.03191402 \mathrm{e}+00$ & $3.19140168 \mathrm{e}-02$ & 1.03 & $1.99966405 \mathrm{e}+00$ & $3.35953571 \mathrm{e}-04$ & 2.05 \\
\hline
\end{tabular}

Table 3. Estimation of $f^{(1)}(x)$ using a non symmetric rule. 


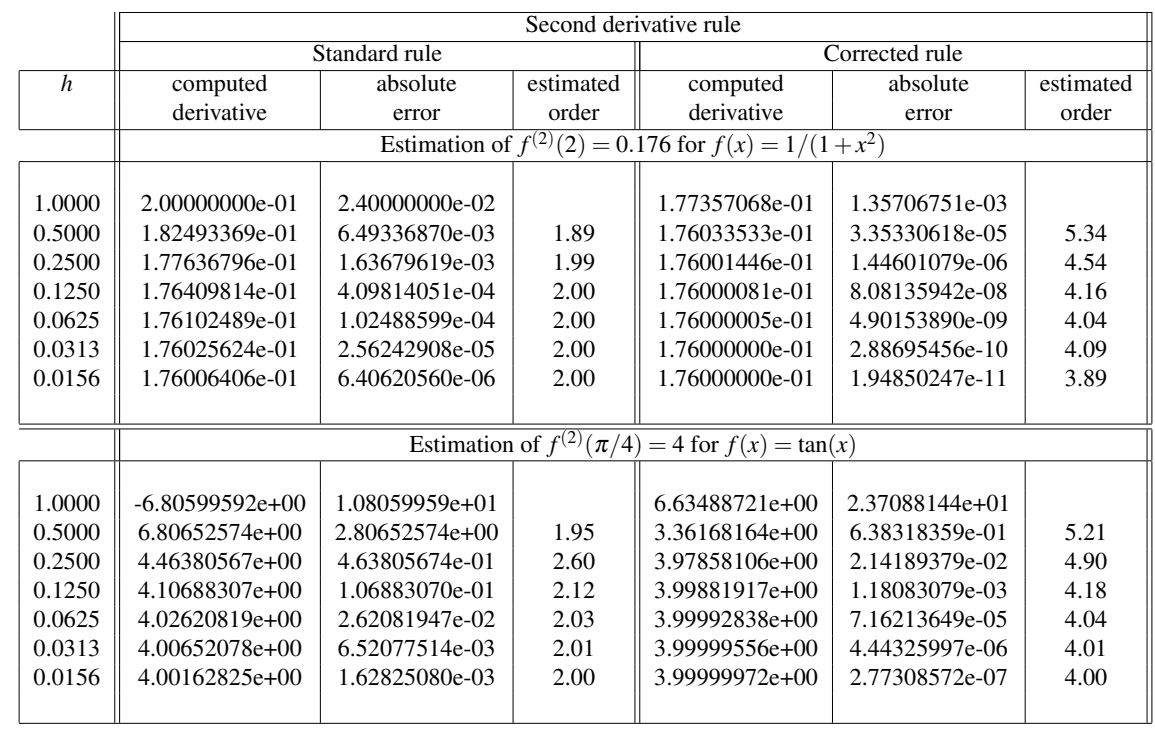

Table 4. Estimation of $f^{(2)}(x)$ using a 3-points symmetric rule.

\begin{tabular}{|c|c|c|c|c|c|c|}
\hline \multirow[b]{3}{*}{$h$} & \multicolumn{6}{|c|}{ Second derivative rule } \\
\hline & \multicolumn{3}{|c|}{ Standard rule } & \multicolumn{3}{|c|}{ Corrected rule } \\
\hline & $\begin{array}{l}\text { computed } \\
\text { derivative }\end{array}$ & $\begin{array}{c}\text { absolute } \\
\text { error }\end{array}$ & $\begin{array}{l}\text { estimated } \\
\text { order }\end{array}$ & $\begin{array}{l}\text { computed } \\
\text { derivative }\end{array}$ & $\begin{array}{c}\text { absolute } \\
\text { error }\end{array}$ & $\begin{array}{l}\text { estimated } \\
\text { order }\end{array}$ \\
\hline & \multicolumn{6}{|c|}{ Estimation of $f^{(2)}(2)=0.176$ for $f(x)=1 /\left(1+x^{2}\right)$} \\
\hline 1.0000 & $2.02636917 \mathrm{e}-01$ & $2.66369168 \mathrm{e}-02$ & & $1.78991046 \mathrm{e}-01$ & $2.99104605 \mathrm{e}-03$ & \\
\hline 0.5000 & $1.83214004 \mathrm{e}-01$ & $7.21400359 \mathrm{e}-03$ & 1.88 & $1.76072351 \mathrm{e}-01$ & $7.23506264 \mathrm{e}-05$ & 5.37 \\
\hline 0.2500 & $1.77818622 \mathrm{e}-01$ & $1.81862247 \mathrm{e}-03$ & 1.99 & $1.76003075 \mathrm{e}-01$ & $3.07542484 \mathrm{e}-06$ & 4.56 \\
\hline 0.1250 & $1.76455347 \mathrm{e}-01$ & $4.55346643 \mathrm{e}-04$ & 2.00 & $1.76000171 \mathrm{e}-01$ & $1.70955263 \mathrm{e}-07$ & 4.17 \\
\hline 0.0625 & $1.76113876 \mathrm{e}-01$ & $1.13876080 \mathrm{e}-04$ & 2.00 & $1.76000010 \mathrm{e}-01$ & $1.03522543 \mathrm{e}-08$ & 4.05 \\
\hline \multirow[t]{2}{*}{0.0313} & $1.76028471 \mathrm{e}-01$ & $2.84714255 \mathrm{e}-05$ & 2.00 & $1.76000001 \mathrm{e}-01$ & $6.04290118 \mathrm{e}-10$ & 4.10 \\
\hline & \multicolumn{6}{|c|}{ Estimation of $f^{(2)}(\pi / 4)=4$ for $f(x)=\tan (x)$} \\
\hline 1.0000 & $-8.26975149 \mathrm{e}+00$ & $1.22697515 \mathrm{e}+01$ & & $7.03551181 \mathrm{e}+00$ & $5.89830241 \mathrm{e}+01$ & \\
\hline 0.5000 & $7.13309802 \mathrm{e}+00$ & $3.13309802 \mathrm{e}+00$ & 1.97 & $2.60314120 \mathrm{e}+00$ & $1.39685880 \mathrm{e}+00$ & 5.40 \\
\hline 0.2500 & $4.51592823 \mathrm{e}+00$ & $5.15928230 \mathrm{e}-01$ & 2.60 & $3.95439864 \mathrm{e}+00$ & $4.56013565 \mathrm{e}-02$ & 4.94 \\
\hline 0.1250 & $4.11879260 \mathrm{e}+00$ & $1.18792597 \mathrm{e}-01$ & 2.12 & $3.99750194 \mathrm{e}+00$ & $2.49806479 \mathrm{e}-03$ & 4.19 \\
\hline 0.0625 & $4.02912227 \mathrm{e}+00$ & $2.91222737 \mathrm{e}-02$ & 2.03 & $3.99984872 \mathrm{e}+00$ & $1.51279188 \mathrm{e}-04$ & 4.05 \\
\hline 0.0313 & $4.00724543 e+00$ & $7.24543362 \mathrm{e}-03$ & 2.01 & $3.99999062 \mathrm{e}+00$ & $9.38143057 \mathrm{e}-06$ & 4.01 \\
\hline
\end{tabular}

Table 5. Estimation of $f^{(2)}(x)$ using a 4-points symmetric rule. 


\begin{tabular}{|c|c|c|c|c|c|c|}
\hline & \multicolumn{6}{|c|}{ Third derivative rule } \\
\hline & \multicolumn{3}{|c|}{ Standard rule } & \multicolumn{3}{|c|}{ Corrected rule } \\
\hline & $\begin{array}{l}\text { computed } \\
\text { derivative }\end{array}$ & $\begin{array}{c}\text { absolute } \\
\text { error }\end{array}$ & $\begin{array}{l}\text { estimated } \\
\text { order }\end{array}$ & $\begin{array}{l}\text { computed } \\
\text { derivative }\end{array}$ & $\begin{array}{c}\text { absolute } \\
\text { error }\end{array}$ & $\begin{array}{c}\text { estimated } \\
\text { order }\end{array}$ \\
\hline & \multicolumn{6}{|c|}{ Estimation of $f^{(3)}(2)=-2.30400000 e-01$ for $f(x)=1 /\left(1+x^{2}\right)$} \\
\hline 1.0000 & $-2.40973631 \mathrm{e}-01$ & $1.05736308 \mathrm{e}-02$ & & $-2.37051773 e-01$ & $6.65177254 \mathrm{e}-03$ & \\
\hline 0.5000 & $-2.34696213 e-01$ & $4.29621292 \mathrm{e}-03$ & 1.30 & $-2.30683859 \mathrm{e}-01$ & $2.83859197 \mathrm{e}-04$ & 4.55 \\
\hline 0.2500 & $-2.31550154 \mathrm{e}-01$ & $1.15015437 \mathrm{e}-03$ & 1.90 & $-2.30415897 e-01$ & $1.58974761 \mathrm{e}-05$ & 4.16 \\
\hline 0.1250 & $-2.30691910 \mathrm{e}-01$ & $2.91909546 \mathrm{e}-04$ & 1.98 & $-2.30400966 \mathrm{e}-01$ & $9.65795639 \mathrm{e}-07$ & 4.04 \\
\hline \multirow[t]{2}{*}{0.0625} & $-2.30473245 \mathrm{e}-01$ & $7.32447324 \mathrm{e}-05$ & 1.99 & $-2.30400059 \mathrm{e}-01$ & $5.94454010 \mathrm{e}-08$ & 4.02 \\
\hline & \multicolumn{6}{|c|}{ Estimation of $f^{(3)}(\pi / 4)=16$ for $f(x)=\tan (x)$} \\
\hline 1.0000 & $-3.06825876 \mathrm{e}+01$ & $4.66825876 \mathrm{e}+01$ & & $-3.90107225 \mathrm{e}-01$ & $1.89206795 \mathrm{e}+02$ & \\
\hline 0.5000 & $2.80069421 \mathrm{e}+01$ & $1.20069421 \mathrm{e}+01$ & 1.96 & $1.14873564 \mathrm{e}+01$ & $4.51264360 \mathrm{e}+00$ & 5.39 \\
\hline 0.2500 & $1.79802007 \mathrm{e}+01$ & $1.98020069 \mathrm{e}+00$ & 2.60 & $1.58535764 \mathrm{e}+01$ & $1.46423628 \mathrm{e}-01$ & 4.95 \\
\hline 0.1250 & $1.64561085 \mathrm{e}+01$ & $4.56108524 \mathrm{e}-01$ & 2.11 & $1.59919858 \mathrm{e}+01$ & $8.01418527 \mathrm{e}-03$ & 4.19 \\
\hline 0.0625 & $1.61118262 \mathrm{e}+01$ & $1.11826164 \mathrm{e}-01$ & 2.02 & $1.59995148 \mathrm{e}+01$ & $4.85235909 \mathrm{e}-04$ & 4.05 \\
\hline
\end{tabular}

Table 6. Estimation of $f^{(3)}(x)$ using a 4-points symmetric rule.

\begin{tabular}{|c|c|c|c|c|c|c|}
\hline \multirow{3}{*}{$h$} & \multicolumn{6}{|c|}{ Fourth derivative rule } \\
\hline & \multicolumn{3}{|c|}{ Standard rule } & \multicolumn{3}{|c|}{ Corrected rule } \\
\hline & $\begin{array}{l}\text { computed } \\
\text { derivative }\end{array}$ & $\begin{array}{c}\text { absolute } \\
\text { error }\end{array}$ & $\begin{array}{c}\text { estimated } \\
\text { order }\end{array}$ & $\begin{array}{l}\text { computed } \\
\text { derivative }\end{array}$ & $\begin{array}{c}\text { absolute } \\
\text { error }\end{array}$ & $\begin{array}{l}\text { estimated } \\
\text { order }\end{array}$ \\
\hline & \multicolumn{6}{|c|}{ Estimation of $f^{(4)}(2)=3.14880000 e-01$ for $f(x)=1 /\left(1+x^{2}\right)$} \\
\hline 1.0000 & $2.80106101 \mathrm{e}-01$ & $3.47738992 \mathrm{e}-02$ & & $3.38842477 \mathrm{e}-01$ & $2.39624769 \mathrm{e}-02$ & \\
\hline 0.5000 & $3.10820640 \mathrm{e}-01$ & $4.05935957 \mathrm{e}-03$ & 3.10 & $3.16060349 \mathrm{e}-01$ & $1.18034893 \mathrm{e}-03$ & 4.34 \\
\hline 0.2500 & $3.14107429 \mathrm{e}-01$ & $7.72571418 \mathrm{e}-04$ & 2.39 & $3.14948783 \mathrm{e}-01$ & $6.87831106 \mathrm{e}-05$ & 4.10 \\
\hline 0.1250 & $3.14701263 \mathrm{e}-01$ & $1.78737171 \mathrm{e}-04$ & 2.11 & $3.14884220 \mathrm{e}-01$ & $4.22036637 \mathrm{e}-06$ & 4.03 \\
\hline \multirow[t]{2}{*}{0.0625} & $3.14836205 \mathrm{e}-01$ & $4.37952495 \mathrm{e}-05$ & 2.03 & $3.14880209 \mathrm{e}-01$ & $2.09043622 \mathrm{e}-07$ & 4.34 \\
\hline & \multicolumn{6}{|c|}{ Estimation of $f^{(4)}(\pi / 4)=80$ for $f(x)=\tan (x)$} \\
\hline 1.0000 & $-2.17800347 e+02$ & $2.97800347 \mathrm{e}+02$ & & $-6.13368590 e+02$ & $2.09714234 \mathrm{e}+03$ & \\
\hline 0.5000 & $1.49934084 \mathrm{e}+02$ & $6.99340843 \mathrm{e}+01$ & 2.09 & $4.20050565 \mathrm{e}+01$ & $3.79949435 \mathrm{e}+01$ & 5.79 \\
\hline 0.2500 & $9.13721867 \mathrm{e}+01$ & $1.13721867 \mathrm{e}+01$ & 2.62 & $7.88356148 \mathrm{e}+01$ & $1.16438517 \mathrm{e}+00$ & 5.03 \\
\hline 0.1250 & $8.26110723 \mathrm{e}+01$ & $2.61107229 \mathrm{e}+00$ & 2.12 & $7.99370476 \mathrm{e}+01$ & $6.29523769 \mathrm{e}-02$ & 4.21 \\
\hline 0.0625 & $8.06396706 \mathrm{e}+01$ & $6.39670610 \mathrm{e}-01$ & 2.03 & $7.99961997 \mathrm{e}+01$ & $3.80027294 \mathrm{e}-03$ & 4.05 \\
\hline
\end{tabular}

Table 7. Estimation of $f^{(4)}(x)$ using a 5-points symmetric rule. 